\title{
Assimilation of Heterogeneous Calibration Measurements for the APEX Spectrometer
}

\author{
Johannes W. Kaiser ${ }^{a}$, Daniel Schläpfer ${ }^{a}$, Jason Brazile ${ }^{a}$, Peter Strobl ${ }^{b}$, \\ Michael E. Schaepman ${ }^{a}$, and Klaus I. Itten ${ }^{a}$ \\ ${ }^{a}$ Remote Sensing Laboratories (RSL), University of Zurich, Winterthurerstrasse 190, \\ 8057 Zurich, Switzerland \\ ${ }^{b}$ German Aerospace Center (DLR), Oberpfaffenhofen, 82234 Wessling, Germany
}

\begin{abstract}
The underlying algorithmic architecture of the level 0 to 1 processing of the APEX (Airborne Prism Experiment) imaging spectrometer is presented. This processing step calculates the observed radiances in physical units from the recorded raw digital numbers.

APEX will operate airborne and record radiance in the solar reflected wavelength range. The system is optimized for land applications including limnology, snow, soil, amongst others. The instrument will be calibrated with a flexible setup in a laboratory as well as on-board.

A concept for the dynamic update of the radiance calibration coefficients for the APEX instrument is presented. The time evolution of the coefficients is calculated from the heterogeneous calibration measurements with a data assimilation technique. We propose a Kalman filter for the initial version of the processor.

Additionally, the structure of the instrument model suitable for the analysis of APEX data is developed. We show that this model can be used for the processing of observations as well as for the calculation of calibration coefficients. Both processes can be understood as inverse problems with the same forward model, i.e. the instrument model.
\end{abstract}

Keywords: APEX, imaging spectrometer, calibration, data assimilation, Kalman filter

\section{INTRODUCTION}

The Airborne Prism EXperiment (APEX) is an airborne dispersive pushbroom imaging spectrometer for the hyperspectral observation of ground reflectances. ${ }^{1,2}$ The applications of the observations include the quantitative retrieval of many biogeophysical parameters, ranging from vegetation parameters for natural hazard management $^{3}$ and climate modeling to geological exploration. ${ }^{4}$ APEX is currently being build in a joint Swiss/Belgian project funded through ESA. It will operationally available to the community on a dedicated aircraft by VITO, Belgium, starting from 2005.

The spectrometer records 300 spectral points in the wavelength range 400-2500 nm with a sampling interval of 5 to $10 \mathrm{~nm}$. Two CCDs are used as detectors in the visible and SWIR spectral range, respectively. The ground pixel size ranges from 2 to $5 \mathrm{~m}$ at a flight altitude of 4 to $10 \mathrm{~km}$. Since APEX is a pushbroom instrument all spectral points are simultaneously observed for 1000 across-track pixels.

The absolute calibration of the spectrometer is crucial for the success of quantitative retrievals from APEX observations. Therefore, extensive calibration measurement will be performed during the commissioning as well as the operational phase of the project. The annual calibration measurement in a laboratory, the so-called Calibration Home Base (CHB), at DLR Oberpfaffenhofen, Germany, will be supplemented by on-board calibration measurements during all data acquisitions. Each of the CHB measurements targets the most important calibration coefficients by adapting the measurement setup in a flexible way. The calibration measurements are heterogeneous as different types are available in an irregular time sequence.

Further author information: Send correspondence to J.W.K.: E-mail: johannes@uni-bremen.de 

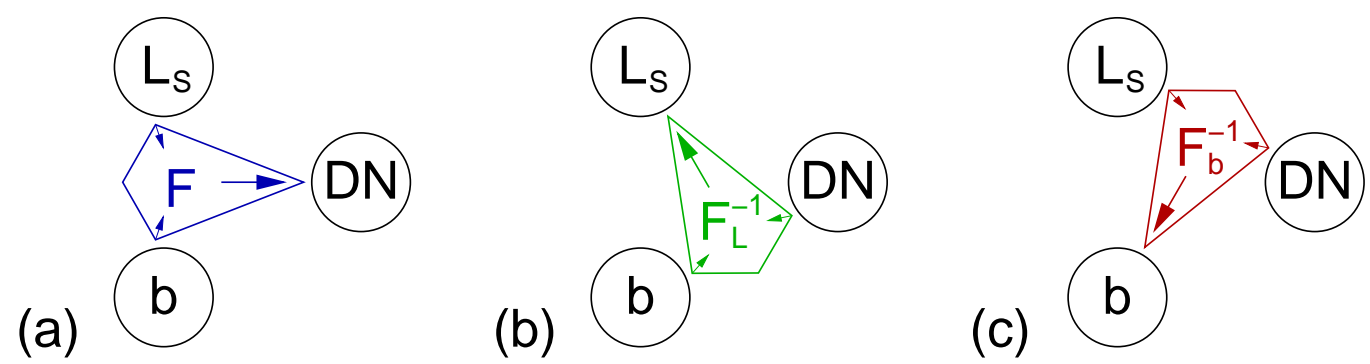

Figure 1. Transformations between radiance at sensor $L_{s}$, recorded raw data $D N$ and calibration coefficients $b$ : (a) data acquisition, (b) level 0 to 1 data processing, (c) calibration coefficient calculation.

The operational Processing and Archiving Facility (PAF) of APEX is currently developed by RSL at the University of Zurich. It performs the transformation of raw data to calibrated level 1 products. This includes the radiometric and optical data calibration and the calculation of the required, time-dependent calibration coefficients from the calibration measurements. The latter cannot be performed in an optimal way by a simple standard procedure because of the heterogeneity of the calibration measurements.

This paper relates the different types of data transformations during data acquisition, data processing and instrument calibration to the underlying instrument model (section 2). The instrument model developed for the APEX PAF is introduced in section 3. The novel calibration coefficient calculation approach of the PAF is described in section 4 .

\section{DATA TRANSFORMATION OVERVIEW}

\subsection{Data Acquisition}

During the data acquisition, the APEX instrument converts the radiance at sensor $L_{s}$ to the digital numbers $D N$ recorded in the raw data:

$$
D N=f\left(L_{s}\right)+\epsilon,
$$

where $f$ denotes the instrument function and $\epsilon$ is the measurement noise. The instrument function is not known exactly. However, it may be approximated with an appropriate instrument model $F$ :

$$
D N=F\left(L_{s}, b, c\right)+\epsilon,
$$

where $b$ denotes the calibration coefficients, $c$ are known instrument settings, and $\epsilon$ is an error term including measurement noise and the model error. Thus the raw digital numbers $D N$ can theoretically be calculated from the radiance at sensor $L_{s}$ and the calibration coefficients $b$ by the instrument model $F$. This is visualized in Fig. 1(a).

\subsection{Data Processing}

The processing in the PAF calculates the original radiance at sensor $L_{s}$ from the raw digital numbers $D N$ and the calibration coefficients $b$, cf. Fig. 1(b). It involves exactly the same quantities as modeling the data acquisition with (2), where they are related by the instrument model $F$. Solving (2) for $L_{s}$ formally yields

$$
L_{s}=F_{L_{s}}^{-1}(D N, b, c)
$$

where the raw data $D N$, calibration coefficients $b$ and settings $c$ are required as input to the inverse $F_{L_{s}}^{-1}$ w.r.t. the radiance $L_{s}$ of the instrument model.

(3) may be interpreted as the inverse problem of solving (2) with the known forward model $F$ for the radiance $L_{s}$. The inverse theory provides methods to find solutions to such problems without determining the inverse $F_{L_{s}}^{-1}$ explicitly. Even if the problem is unstable the problem may be regularized by introducing apriori information.

However, $F_{L_{s}}^{-1}$ might be known explicitly. In this case (3) can also be used directly to calculate the radiance $L_{s}$. In any case an accurate, yet as simple as possible, instrument model $F$ is needed as forward model for the development of the data processing algorithms. 


\subsection{Instrument Calibration}

The calibration coefficients $b$ are determined from the digital numbers $D N$ and the radiance at sensor $L_{s}$ of the calibration measurements, cf. Fig. $1(\mathrm{c}) . D N$ is recorded in the raw data while $L_{s}$ is produced in a well-defined form by the calibration equipment. Thus the same quantities as in the modeling (2) of the data acquisition and in the processing (3) are involved. Again, they are related by the instrument model $F$. Solving (2) for $b$ formally yields the desired calibration coefficients

$$
b=F_{b}^{-1}\left(L_{s}, D N, c\right)
$$

where the radiance at sensor $L_{s}$, raw data $D N$, and instrument settings $c$ are required as input to the inverse $F_{b}^{-1}$ w.r.t. the calibration coefficients $b$ of the instrument model $F$.

Analogously to the interpretation of (3), (4) may be interpreted as inverse problem and solved either explicitly or with the methods of the inverse theory. Again, the instrument model $F$ is needed.

\section{INSTRUMENT MODEL}

\subsection{Forward Model of Data Acquisition}

Section 2 has shown that an appropriate instrument model $F$ is of central importance as forward model for solving the inverse problem of data processing as well as that of instrument calibration. Either the mathematical formulation of the instrument model can be inverted explicitly or its implementation can be used as forward model in an inversion algorithm.

The instrument model should reproduce the instrument's behavior accurately while using as few calibration coefficients as possible. The calibration coefficients need not have simple physical interpretations. The overall structure of the transformations which convert the radiance field to digital numbers inside the instrument is shown on the left side of Fig. 2 and summarized below.

In the optics of APEX, the photons of the radiance at sensor $L_{s}$ are distributed on the pixels of the detector. At the same time, the radiance is attenuated by the transmission of the optics and detector. Even though the distribution and the attenuation are a combined process in the instrument, they may be regarded as sequential operations during the processing: The redistribution may be characterized by normalized point spread functions (PSFs) along with ghost and stray light. For such normalized PSFs, the attenuation may subsequently be characterized by scaling factors. The intermediate quantity after redistribution, before scaling shall be called photon flux at sensor $P_{S}$. It turns out to be very useful for the algorithm design even though it does not represent an existing quantity in APEX.

In the detector, the photons are converted to electrons, creating a current. Dark current is added and integration over the time results in an electric charge. During readout of the detector, the smear effect adds to the charge in a way depending on direction of the readout.

When reading out the detector, the charges are converted to voltages, which are subsequently scaled in the analog amplifier and finally converted to the digital numbers $D N$ of the raw data in the analog-digital-converter $(\mathrm{ADC})$.

A more detailed description of the APEX PAF instrument model is given by Schläpfer et al.. ${ }^{5}$

\subsection{Inverse Model for Data Processing}

The processing of the PAF aims at inverting the transformations by the instrument introduced in section 3.1 and Fig. 2. This is equivalent to the application of the inverse $F_{L_{s}}^{-1}$ w.r.t. the radiance of the instrument model $F$.

The transformation can be split into two fundamentally different types. Thus the the algorithm development is divided into the following four steps, cf. Fig. 2, right side:

Radiometric Data Calibration The transformations from photon flux at sensor $P_{S}$ to digital numbers $D N$ can be parameterized as a sequence of scalings and additions of offsets. They act on quantities associated to individual detector pixels. (Only the smear effect depends the radiance in other detector pixels.) Since these transformations are well-characterized they can be inverted explicitly. 


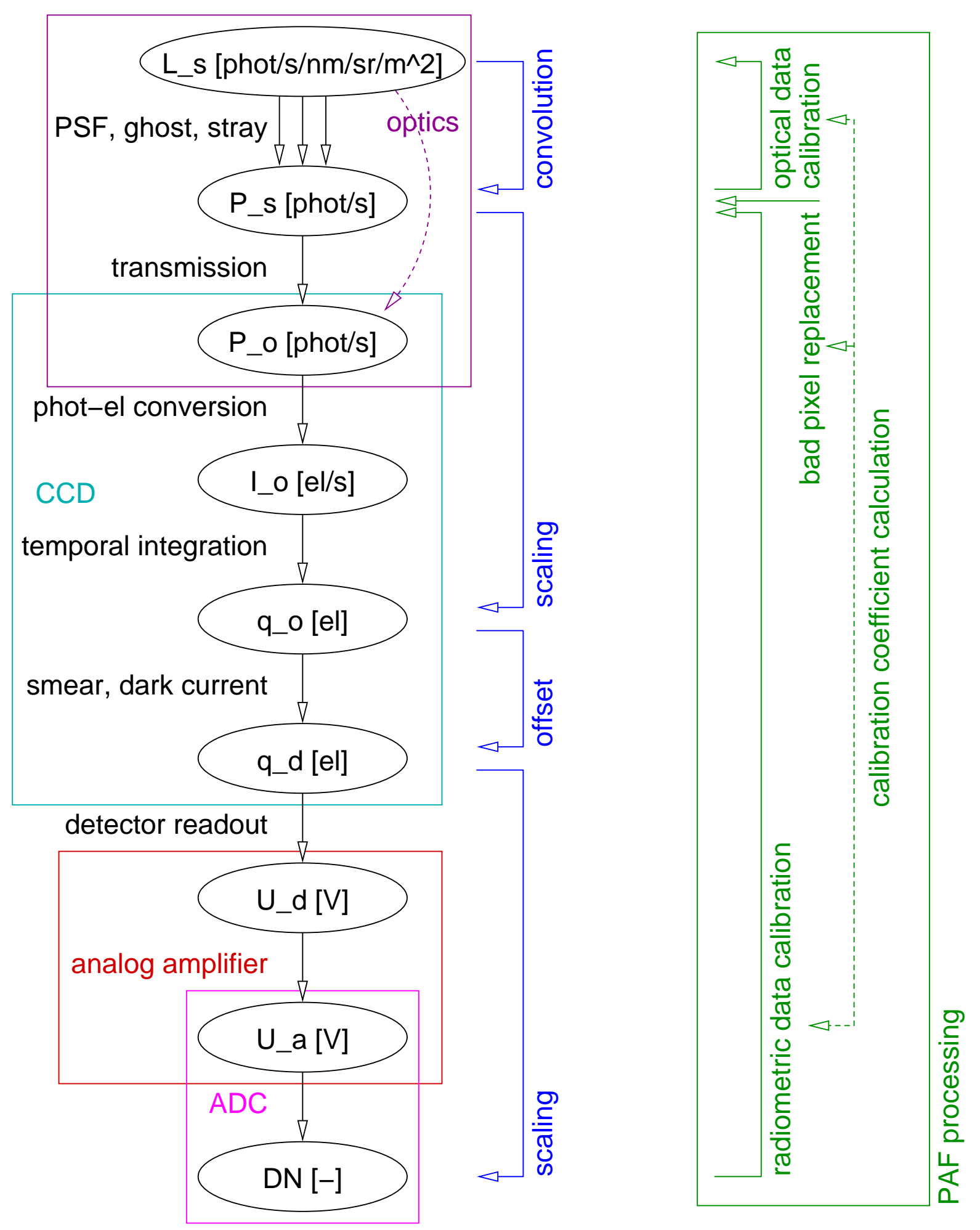

Figure 2. Data transformations in the APEX instrument (left) and the APEX PAF (right). 
Optical Data Calibration The PSF convolution principally cannot be inverted without additional constraints, e.g. demanding smoothness. Thus the deconvolution would introduce a priori information into the data product. It needs to be evaluated carefully, how much contamination with a priori information can be tolerated.

The PSF convolution is intimately linked to the spectral and spatial, i.e. angular, calibration of the data. In this context the smile and frown correction is performed by an appropriate data resampling in the spectral and across-track dimensions.

Additionally, ghost- and stray light corrections seem necessary. The required algorithms are defined as part of the "Optical Data Calibration" procedure.

Bad Pixel Replacement Bad and dead pixels exist on the detector. Since most resampling algorithms require data on a regular grid the missing values are substituted with interpolated ones after the radiometric data calibration.

Calibration Coefficient Calculation The calibration coefficients for the three processing steps defined above need to be calculated from the calibration measurements. A suitable algorithm for this calculation is defined. The complete set of calibration measurements is used to calculate appropriate time-dependent calibration coefficients for the radiometric and optical data calibration steps.

Detailed descriptions of the APEX PAF data processing algorithms are given in Schläpfer et al. ${ }^{5}$

\section{CALIBRATION COEFFICIENTS CALCULATION}

\subsection{Task}

According to section 2.2 the calibration coefficients $b$ are required as input for the data processing. The best estimates of the values of the calibration coefficients $b$ at each instant in time needs to be calculated from the calibration measurements by the PAF. The parameters summarized in Table 1 are required. ${ }^{5}$ All parameters ultimately need to be known for each pixel. However, the wavelength $\lambda$ and across-track angle $\theta$ are not treated explicitly. Instead they are described by function parameters $a_{i}^{j}\left(i=1 \ldots N^{j}\right)$ and $a_{i}^{k}\left(i=1 \ldots N^{k}\right)$. Thus the goal is to calculate the time-dependent vector of all calibration coefficients

$$
b(t) \equiv\left(\begin{array}{c}
\left(G^{1,1}(t) \cdots G^{1, N^{k}}(t)\right)^{T} \\
\vdots \\
\left(G^{N^{j}, 1}(t) \cdots G^{N^{j}, N^{k}}(t)\right)^{T} \\
\left(d c^{1,1}(t) \cdots d c^{1, N^{k}}(t)\right)^{T} \\
\vdots \\
\left(d c^{N^{j}, 1}(t) \cdots d c^{N^{j}, N^{k}}(t)\right)^{T} \\
\left(a_{1}^{j}(t) \cdots a_{N^{j}}^{j}(t)\right)^{T} \\
\left(a_{1}^{k}(t) \cdots a_{N^{k}}^{k}(t)\right)^{T}
\end{array}\right)
$$

where $N^{j}$ and $N^{k}$ denote the number of detector pixels in wavelength and across-track directions. The dead/bad pixel map is omitted as it is the only parameter of discrete, i.e. logical, type.

\subsection{Available Information}

Two principally different sources of information on the calibration coefficients are available: calibration measurements and apriori insight into the characteristics of the instrument. 
Table 1. List of Calibration Coefficients

\begin{tabular}{llc}
\hline symbol & description & parameters \\
\hline$G^{j, k}$ & gain factor & \\
$d c^{j, k}$ & dark current factor & \\
$d^{j, k}$ & bad/dead pixel map & \\
$\lambda^{j, k}$ & wavelength & $a_{i}^{j}$ \\
$\theta^{j, k}$ & across-track angle & $a_{i}^{k}$ \\
\hline
\end{tabular}

\subsubsection{Observation: Calibration Measurements}

Calibration measurements are performed in the CHB and on-board. The former include spectral, spatial, and radiometric calibration measurements along with stray-light, polarization, and temperature sensitivity measurements and a high altitude window calibration. ${ }^{6}$

Radiometric calibration with an internal reference light source and spectral calibrations with rare-earth doted filters is performed using the in-flight calibration facility during each deployment of APEX. ${ }^{7}$ These measurements increase the heterogeneity of the calibration observations even further. They are initially used as a reference for monitoring the calibration based on the CHB. If necessary they may be included in the actual calculation of the calibration coefficients later on.

\subsubsection{System Insight}

Knowledge about the design of APEX provides additional information about relations between the calibration coefficients and about their evolution in time. For example, the parameterizations of $\lambda^{j, k}$ and $\theta^{j, k}$ are one way of incorporating this information usefully. ${ }^{5}$ Additionally, correlations between the calibration coefficients may be described in the calibration coefficient covariance matrix. Information on the time evolution can be accounted for in the system model, see below.

\subsection{Basic Algorithm Layout}

A flexible data assimilation (DA) algorithm is developed in order to combine the information from all of the heterogeneous calibration measurements as well as from the system insight in an optimal way.

Basic components of all DA algorithms are illustrated in Fig. 3 for the example of a Kalman filter: The initial measurement of the system is analyzed with the observation operator $H$. It yields the observation $b_{o}$ along with its uncertainty $\sigma_{o}$. The values are generally interpreted as mean and standard deviation of a Gaussian probability density distribution. The observation is combined in a consistent, optimal way with any a priori knowledge $b_{b}$ with $\sigma_{b}$. If no a priori knowledge is available then $\sigma_{b}=\infty$ and the observed state is identical to the analysis $b_{a}$ with $\sigma_{a}$. Subsequently, its time evolution is calculated with the system model $M$ until the next observation is available. Thus the background $b_{b}$ with $\sigma_{b}$ is obtained. Since no information on the system is available during this time $\sigma_{a}<\sigma_{b}$ usually holds. The time evolution is shown as dark blue dashed line in Fig. 3.

When a new observation $b_{o}$ with $\sigma_{o}$ is available it is combined in a consistent, optimal way with the background $b_{b}$ with $\sigma_{b}$. Thus a new analysis $b_{a}$ with $\sigma_{a}$ is obtained. $\sigma_{a} \leq \sigma_{b}$ and $\sigma_{a} \leq \sigma_{o}$ hold. They reflect the increase in knowledge resulting from combining several sources of information. The procedure is repeated from this point onwards: calculation of time evolution, combination with new observation, ...

At every instance in time the Kalman filter is combining the past observations in an optimal way. An interpolation between the analysis $b_{a}$ would yield a better approximation to the true values, compare the light green dashed line in Fig. 3. This approach requires that calibration coefficient observations taken after the instance in time under consideration are also available. More sophisticated approaches like the so-called Kalman smoother ${ }^{8}$ exist for this case.

Since the system is described by a large set of calibration coefficients the uncertainty is described by a covariance matrix $S$ instead of the uncertainty $\sigma$, cf. appendix A. 

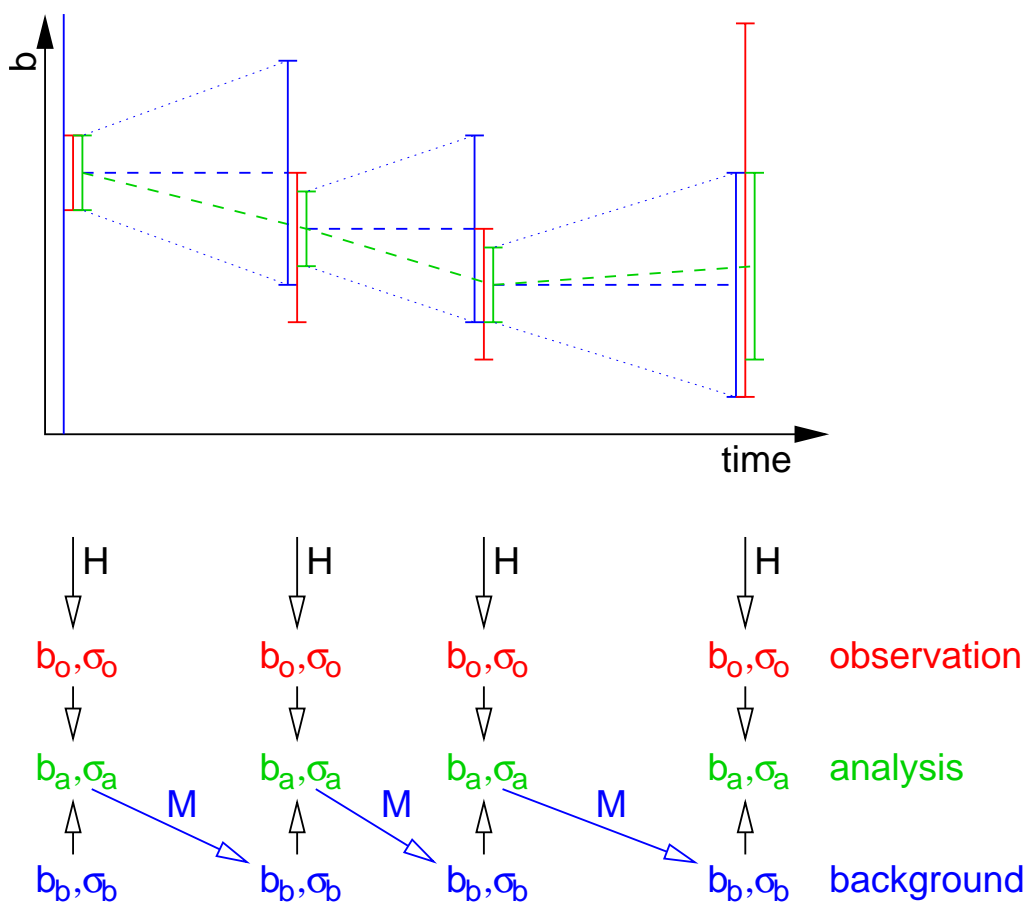

Figure 3. Illustration of a 1-d Kalman filter.

The following sections discuss the designs of components mentioned above for the APEX PAF. (The employed covariance matrix and the optimal combination are detailed in appendices A and B.)

\subsection{Observation Operator $H$}

The calibration coefficients are determined during calibration measurements in the CHB. This data transformation has been introduced in section 2.3 and Fig. 1(c). In order to derive the calibration coefficients $b_{o}$ the observation operator $H$ can be implemented as the inverse of the instrument model $F$ w.r.t. the calibration coefficients $b$, cf. (4).

Additionally, the associated covariance matrix $S_{o}$ needs to be provided. It can also be calculated within the framework of an inverse method if the covariance matrices of the input variables $L_{s}$ and $D N$ are provided. Thus, the calibration coefficients are provided to the PAF as the vector $b_{o}$ with associated covariance matrix $S_{o}$.

\subsection{System Model}

The system model $H$ describes the time evolution of the calibration coefficients $b$ and their covariance matrix $S$.

The calibration coefficients evolution is assumed to be a Markov process, i.e. $b$ depends only on that of the previous time step and a random component $\epsilon$. This view assumes that known dependencies like the one of the dark current on temperature are suitably parameterized.

A Markov process with constant time evolution of parameters and linear increase of uncertainty is used in the processor:

$$
\begin{aligned}
b\left(t_{2}\right) & =b\left(t_{1}\right)+\epsilon, \\
S\left(t_{2}\right) & =S\left(t_{1}\right) \times\left(1+\frac{t_{2}-t_{1}}{\delta}\right), \\
\text { with } \delta & >0 .
\end{aligned}
$$


Since all elements of $S$ are scaled with the same factor $>1$ in (7) the correlations are preserved during the time evolution, cf. (9). Thus the system insight included in the covariance matrix is preserved while the overall knowledge of $b$ decreases: the variance doubles within the time interval $\delta$.

The calibration coefficient time evolution (6) and the time constant $\delta$ should be adjusted to the observed behavior during the entire life time of the instrument.

\subsection{Data Assimilation Scheme}

Data assimilation (DA) algorithms have been developed in several areas of Earth observation. The most prominent are:

numerical weather prediction Some specifics are a very large state vector, a relatively good system model and high sensitivity to the initial state as the system exhibits deterministic chaos. Therefore, much of the work aims at determining the initial state as accurately as possible with variational DA schemes. A crucial point is finding a suitable approximation for the state's covariance matrix, which is too large to be stored and calculated explicitly. ${ }^{9,10}$

chemical modeling Models of the atmospheric chemistry do generally not display chaotic behavior. Instead their weak points often lie in the poor knowledge of many system model parameters, e.g. reaction rate constants. DA is used to overcome this weakness while determining the atmospheric concentration from observations. Another application is to derive knowledge on species that are not observed themselves. ${ }^{11,12}$

remote sensing Both areas mentioned above assimilate remote sensing data into atmospheric models. A comprehensive description of atmospheric remote sensing theory and its link to DA is given by. ${ }^{8}$

Efforts to apply DA to instrument calibration in Earth observation are rather limited: Just one study by Freedman et al. ${ }^{13}$ regarding the calibration of Landsat 7 is known to the authors.

The choice of a suitable DA scheme depends largely on the system model:

- If the model is poor then the assumption of a Markov process, i.e. random influences, is appropriate. Then a sequential approach like the Kalman filter/smoother would be suitable.

- If the model is well known but it is very sensitive to the system state then a variational scheme can be successfully applied.

- If the model is well-known but its input parameters are poorly known then the parameters may be determined with a variational approach.

- If the model is well-known but its input parameters are variable then a DA for the input parameters should be implemented.

Since APEX is relatively stable, but little else is known about the system, a Kalman filter is implemented in the first version of the PAF. This corresponds to the calibration coefficient evolution given as dark blue dashed line in Fig. 3.

During the continued operations of APEX, calibration measurements that take place after the data acquisitions become available. This information will be used for reprocessing the observations with improved calibration coefficients obtained with a Kalman smoother. The improved calibration coefficients correspond to the light green dashed line in Fig. 3.

The Kalman filter and smoother are sequential DA schemes. During the life time of APEX an advanced parameterization for the system model may be established. Subsequently a variational DA scheme can be used for optimizing these parameters. 


\section{CONCLUSIONS}

The airborne spectrometer APEX will record hyperspectral ground observation data. Quantitative product generation will be possible with excellent accuracy as the instrument calibration is given a high priority in the project. It is facilitated by extensive calibration measurements both in the laboratory and on-board. However, the heterogeneity of these measurements poses a challenge to the data processing chain.

We propose a novel data assimilation scheme for the dynamic calculation of the calibration coefficients and their time dependency. Thus an optimal combination of all information from the various calibration measurement plus any apriori considerations is achieved.

We have also shown that an accurate, yet as simple as possible instrument model facilitates the processing of target observations as well as the calculation of calibration coefficients. In both cases the same instrument model is employed as forward model in an inversion algorithm. Thus consistency throughout the entire data processing of APEX is achieved.

Finally, the basic layout of the required instrument model for APEX has been derived.

\section{APPENDIX A. COVARIANCE MATRIX}

Any covariance matrix $S$ of dimension $n \times n$ has the general form

$$
S=\left(\begin{array}{cccc}
\sigma_{1}^{2} & c_{1,2} \sigma_{1} \sigma_{2} & \cdots & c_{1, n} \sigma_{1} \sigma_{n} \\
c_{2,1} \sigma_{2} \sigma_{1} & \sigma_{2}^{2} & \cdots & c_{2, n} \sigma_{2} \sigma_{n} \\
\vdots & \vdots & \ddots & \vdots \\
c_{n, 1} \sigma_{n} \sigma_{1} & c_{n, 2} \sigma_{n} \sigma_{2} & \cdots & \sigma_{n}^{2}
\end{array}\right),
$$

where $\sigma_{i}^{2}$ is the variance of the $i$ th element and $c_{i, j} \in[-1,1]$ is the correlation between the $i$ th and $j$ th elements. The covariance matrix evidently is square and symmetric.

According to present understanding of APEX the different calibration coefficients listed in Table 1 exhibit no cross-correlation, e.g. the gain factor of a pixel is not linked to its dark current factor. Therefore, the covariance matrix can always be written in a block-diagonal form.

Only the wavelength $\lambda^{j, k}$ and across-track angle $\theta^{j, k}$ vary systematically across the detector. This systematic behavior can be used to derive the correlations between, e.g., the wavelength associated with adjacent pixels. In the case of APEX the systematic behavior is exploited to replace the large set of parameters $\lambda^{j, k}$ and $\theta^{j, k}$ with a smaller set of parameters $a_{i}^{j}$ and $a_{i}^{k}$. Since no correlation between the latter set of parameters is known the covariance matrix is actually diagonal.

The covariance matrices $S$ possibly have to represent "no knowledge". Since the corresponding infinite diagonal elements $\sigma_{i}^{2}$ cannot be store in conventional computer programming language variables the covariance matrices' inverses $S^{-1}$ are stored instead.

\section{APPENDIX B. OPTIMAL COMBINATION}

The combination of two scalar measurement $x_{o}$ and $x_{b}$ with variances $\sigma_{o}^{2}$ and $\sigma_{b}^{2}$ is familiar from basic text books:

$$
\begin{aligned}
x_{a} & =\sigma_{a}^{2}\left(\frac{1}{\sigma_{o}^{2}} x_{o}+\frac{1}{\sigma_{b}^{2}} x_{b}\right) \\
\text { with } \sigma_{a}^{2} & =\frac{1}{1 / \sigma_{o}^{2}+1 / \sigma_{b}^{2}} .
\end{aligned}
$$

Analogously, two observations of vectors $b_{o}$ and $b_{b}$ with covariance matrices $S_{o}$ and $S_{b}$ are combined by

$$
\begin{aligned}
b_{a} & =S_{a}\left(S_{o}^{-1} b_{o}+S_{b}^{-1} b_{b}\right) \\
\text { with } S_{a} & =\left(S_{o}^{-1}+S_{b}^{-1}\right)^{-1},
\end{aligned}
$$


see, e.g., Rodgers. ${ }^{8}$ These equations describe the behavior of multidimensional Gaussian probability density functions.

The multidimensional case formally involves the inversion of a matrix. This step needs careful implementation in order to avoid numerical instability and computational inefficiency. Additionally, the existence of the inverse may need checked.

\section{ACKNOWLEDGMENTS}

The work in this paper is being carried out under ESA/ESTEC contract no. 1449/01/NL/Sfe. The support of the University of Zurich is acknowledged.

\section{REFERENCES}

1. K. Itten, M. Schaepman, L. De Vos, L. Hermans, H. Schlaepfer, and F. Droz, "Apex-airborne prism experiment a new concept for an airborne imaging spectrometer," in Proceedings of the Third International Airborne Remote Sensing Conference and Exhibition, Vol. I, pp. 181-188, ERIM International Inc., (Copenhagen (DK)), 1997.

2. M. Schaepman and et.al., "Apex: current status of the airborne dispersive pushbroom imaging spectrometer," in Sensors, Systems, and Next Generation Satellites IX, H. S. R. Meynart, S.P. Neeck, ed., this issue, SPIE, (Barcelona), 2003.

3. B. Kötz, M. Schaepman, F. Morsdorf, P. Bowyer, K. Itten, and B. Allgöwer, "Radiative transfer modeling within a heterogeneous canopy for estimation of rorest fire fuel properties," Remote Sensing of Environment , 2003. submitted.

4. R. O. Green, M. L. Eastwood, C. M. Sarture, T. G. Chrien, M. Aronsson, B. J. Chippendale, J. A. Faust, B. E. Pavri, C. J. Chovit, M. S. Solis, M. R. Olah, and O. Williams, "Imaging spectroscopy and the airborne visible infrared imaging spectrometer (aviris)," Remote Sensing of Environment 65(3), pp. 227-248, 1998.

5. D. Schläpfer, J. Kaiser, J. Brazile, M. Schaepman, and K. Itten, "Calibration concept for potential optical aberrations of the apex pushbroom imaging spectrometer," in Sensors, Systems, and Next Generation Satellites IX, H. S. R. Meynart, S.P. Neeck, ed., this issue, SPIE, (Barcelona), 2003.

6. P. Strobl, W. Mooshuber, M. Schaepman, and D. Schläpfer, Technical Note 5, Calibration Hardware Requirements: Performance and Calibration Requirements for APEX. RSL, University of Zurich, 2001. ESA/ESTEC contract-no. 14906/00/NL/DC.

7. D. Schläpfer, M. Schaepman, S. Bojinski, and A. Börner, "Calibration concept for the airborne prism experiment (APEX)," Can. J. of R. S. 26(5), pp. 455-465, 2000.

8. C. D. Rodgers, Inverse Methods for Atmospheric Sounding: Theory and Practice, vol. 2 of Series on Atmospheric, Oceanic and Planetary Physics, World Scientific, Singapore, New Jersey, London, Hong Kong, 2000 .

9. O. Talagrand, "Assimilation of observations, an introduction," Journal of the Meteorological Society of Japan 75(1B), pp. 191-209, 1997.

10. A. C. Lorenc, S. P. Ballard, R. S. Bell, N. B. Ingleby, P. L. F. Andrews, D. M. Barker, J. R. Bray, A. M. Clayton, T. Dalby, D. Li, T. J. Payne, and F. W. Saunders, "The met. office global three-dimensional variational data assimilation scheme," Quarterly Journal of the Royal Meteorological Society 126(570), pp. 2991-3012, 2000.

11. D. J. Lary, "Data assimilation: a powerful tool for atmospheric chemistry," Philosophical Transactions of the Royal Society of London Series a-Mathematical Physical and Engineering Sciences 357(1763), pp. 34453457, 1999.

12. R. Mueller, H. Bovensmann, J. Kaiser, A. Richter, A. Rozanov, F. Wittrock, and J. Burrows, "Consistent interpretation of ground based and GOME BrO slant column data," Adv. Space Res. 29(11), pp. 1655-1660, 2002.

13. E. Freedman and J. Byrne, "Combining multiple sources for radiometric calibration of Landsat 7 using a kalman filter," IAPRS 30(5W1), 1995. 